\title{
Acute Generalized Exanthematous Pustulosis during Puerperal Period Induced by Azithromycin: Case Report
}

\author{
Janete Vettorazzi 1,2,3*, Edimárlei Gonsales Valério1,2,3, Michele Luz Kayser², \\ Luíza Guazzelli Pezzali³ , Daniela Vanessa Vettori1,3, Gabriela Francoes Rostirolla1,3 \\ ${ }^{1}$ Postgraduate Program in Health Sciences: Gynecology and Obstetrics, Federal University of Rio Grande do Sul (UFRGS), \\ Porto Alegre, Brazil \\ ${ }^{2}$ Department of Gynecology and Obstetrics, Faculty of Medicine (FAMED), Federal University of Rio Grande do Sul (UFRGS), \\ Porto Alegre, Brazil \\ ${ }^{3}$ Service of Gynecology and Obstetrics, Hospital de Clínicas de Porto Alegre (HCPA), Porto Alegre, Brazil \\ Email: *jvettorazzi@hcpa.edu.br
}

How to cite this paper: Vettorazzi, J., Valério, E.G., Kayser, M.L., Pezzali, L.G., Vettori, D.V. and Rostirolla, G.F. (2018) Acute Generalized Exanthematous Pustulosis during Puerperal Period Induced by Azithromycin: Case Report. Open Journal of Obstetrics and Gynecology, 8, 1156-1162. https://doi.org/10.4236/ojog.2018.812117

Received: September 3, 2018

Accepted: October 13, 2018

Published: October 16, 2018

Copyright $\odot 2018$ by authors and Scientific Research Publishing Inc. This work is licensed under the Creative Commons Attribution International License (CC BY 4.0).

http://creativecommons.org/licenses/by/4.0/

\begin{abstract}
Background: The use of antibiotics, especially beta-lactams and macrolides, may be associated with dermatopathies, such as Acute Generalized Exanthematous Pustulosis (AGEP), which is an uncommon cutaneous adverse reaction. Case: We report a case of AGEP, in a 36-year-old multiparous (G5P3C1A1) woman, with 38 weeks of gestation, admitted to the hospital to induce labor. Due to cephalopelvic disproportion, the cesarean section was indicated. In the postoperative period, the patient evolved with cutaneous rash, accompanied by productive cough and dyspnea. Because it was a fever of obscure origin, the treatment with antibiotics, including azithromycin, was initiated. On subsequent days, she presented pustules on the back, abdomen and extremities. Such reaction was attributed to the use of azithromycin. When the drug was discontinued, the lesions regressed significantly. Conclusion: The clinical picture of AGEP may occur with persistent high fever and therefore could be confused with systemic infections, consequently, being treated with wrong medications capable of aggravating the adverse cutaneous reaction, worsening the course of the disease that could be easily treated by stopping the use of the causative drug. This case shows the importance of including AGEP as a differential diagnosis of dermatopathies in the pregnancy-puerperal cycle, especially in women who are using various medications, including antibiotics.
\end{abstract}

\section{Keywords}

Acute Generalized Exanthematous Pustulosis, Dermatopathy, High-Risk 
Gestation, Case Report

\section{Introduction}

Acute Generalized Exanthematous Pustulosis (AGEP) is a rare dermatopathy, with an incidence of 1 - 5 cases/million/year, characterized by acute and extensive emergence of sterile, non-follicular pustules on an erythematous basis. Because it is accompanied by fever and leukocytosis [1] [2], mainly eosinophilia, the differential diagnosis of possible systemic diseases is important in order to avoid inappropriate treatment [3]. In more than $90 \%$ of cases, it is a drug-induced reaction, mostly caused by antibiotics such as beta-lactams and macrolides [4].

The risk of complications, multiple organ involvement and death associated with the disease are greater in elderly patients with other comorbidities, with a mortality rate of less than 5\% [4] [5] [6]. The resolution of the disease occurs between 4 and 15 days after the suspension of the causative drug, followed by scaling of the affected areas [4] [6].

Although it is a rare disease, AGEP is a differential diagnosis with many dermatopathies and can be easily managed and resolved after the correct diagnosis.

The following case report of AGEP occurred in Hospital de Clínicas Porto Alegre (HCPA), a public-private hospital linked to the educational institution Universidade Federal do Rio Grande do Sul (UFRGS), and aims to share the evolution of this experience to be reminded in similar situations.

\section{Case Report}

A 36-years-old caucasian woman, multiparous (G6P3C1A1), with obesity and gestational diabetes mellitus, at 38 weeks of pregnancy, sought care in HCPA obstetric emergency due to loss of fluid, without labor. In the physical examination, the patient had a body mass index of $40.8 \mathrm{~kg} / \mathrm{m}^{2}$, blood pressure $140 / 84$ $\mathrm{mmHg}$ and a proteinuria/creatininuria ratio of 0.54 , therefore diagnosed as mild preeclampsia. The labor was induced with oxytocin, however the patient progressed to cesarean delivery due to cephalopelvic disproportion. Below, the postoperative (PO) evolution is described (Table 1).

Table 1. Clinical evolution.

\begin{tabular}{|c|c|c|}
\hline Post Operative day & Clinical evolution & Medical conduct \\
\hline 2nd & $\begin{array}{l}\text { Patient presented a cutaneous rash in the abdomen and } \\
\text { lower limbs (Figure 1), associated with mild pruritus. }\end{array}$ & $\begin{array}{l}\text { Dexchlorpheniramine. Suspended potential skin-reactive drugs } \\
\text { such as dipyrone and diclofenac. }\end{array}$ \\
\hline $3 \mathrm{rd}$ & $\begin{array}{l}\text { The skin rash remained, now with periumbilical area with } \\
\text { phlogistic signs, without contiguity with operative wound. } \\
\text { Patient also presented desaturation in ambient air }(90 \% \\
\text { capillary oxygen saturation), associated with dyspnea and } \\
\text { productive cough. Tachycardia and subfebrile peak of } \\
\text { axillary temperature }\left(37.7^{\circ} \mathrm{C}\right) \text { were present, with no change } \\
\text { in pulmonary auscultation. }\end{array}$ & $\begin{array}{l}\text { Screening tests for fever of unknown origin were requested, } \\
\text { which revealed left leukocytosis ( } 15.380 \text { leukocytes with } 6 \% \text { of } \\
\text { sticks), increase in C-reactive protein ( } 318.9 \text { ), X-ray with } \\
\text { discrete bilateral diffuse infiltrate and no consolidations } \\
\text { (Figure } 2 \text { ). Therefore, empirical use of antibiotic combination } \\
\text { with piperacillin, tazobactan and azithromycin was considered } \\
\text { due to possible infectious disease of unknown focus. }\end{array}$ \\
\hline
\end{tabular}




\section{Continued}

4 th

6 th peaks and the appearance of pustules in abdomen and lower limbs was verified (Figure 3 ).

6 th of axillary temperature between $38^{\circ} \mathrm{C}$ and $39^{\circ} \mathrm{C}$.

Ambulatory segment. Progressive improvement of skin lesions. No fever episodes. Operative wound in second
Despite the antibiotic therapy, patient maintained febrile

Onset of lesions on the back. Patient presented febrile peaks The antimicrobial was maintained and a consulting for the

Dermatology team was requested.

Abdominal ultrasound revealed a heterogeneous net formation located in intra-abdominal topography, interposed between the anterior abdominal wall and the uterus $(8.8 \times 1.5$ $\times 8.5 \mathrm{~cm}$ ), with possibility of postoperative blood collection. C-reactive protein remained high (285.8).

Exploratory laparotomy: purulent secretion was identified from the subcutaneous tissue to the aponeurosis. Abdominal cavity without collections or active bleeding. Cavity and subcutaneous lavage was carried out. Bacteriological subcutaneous collection was requested, showing absence of bacterial growth. A biopsy of the skin lesions was performed. The diagnostic hypothesis was AGEP and the use of Azithromycin suspended.

Patient was kept on Piperacillin and Tazobactan for 10 days after reintervention, being discharged on the 18th postoperative day, remained afebrile and with an operative wound in second intention healing.

Ambulatory return in 1 week, patient did not attend. intention healing.

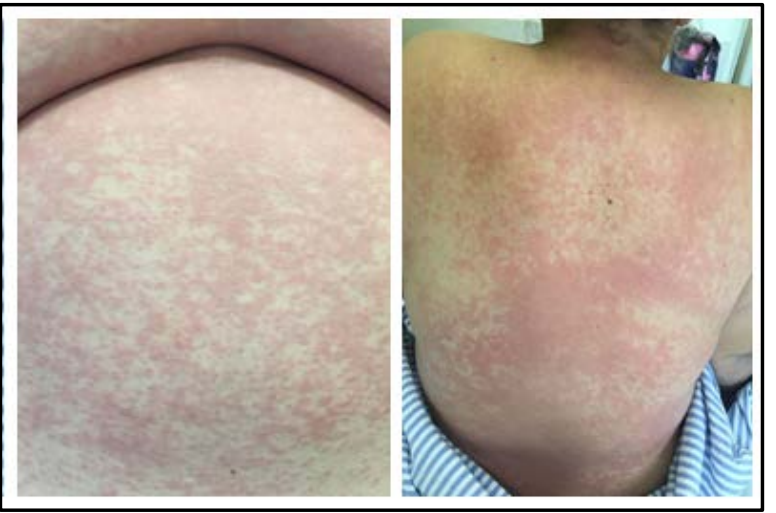

Figure 1. Skin rash on the 2nd PO.

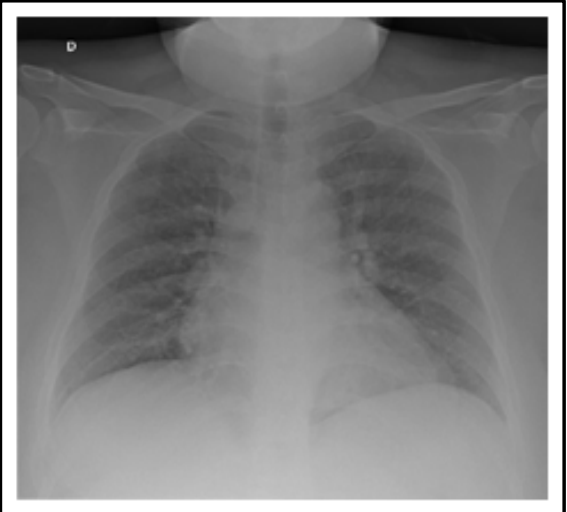

Figure 2. Bilateral interstitial infiltrate on the 3rd PO. 


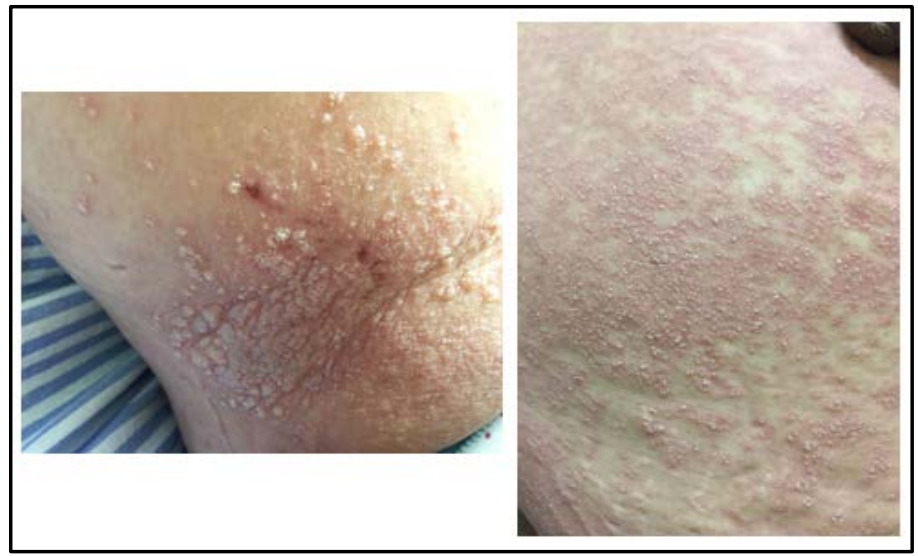

Figure 3. Pustular lesions on the 6th PO.

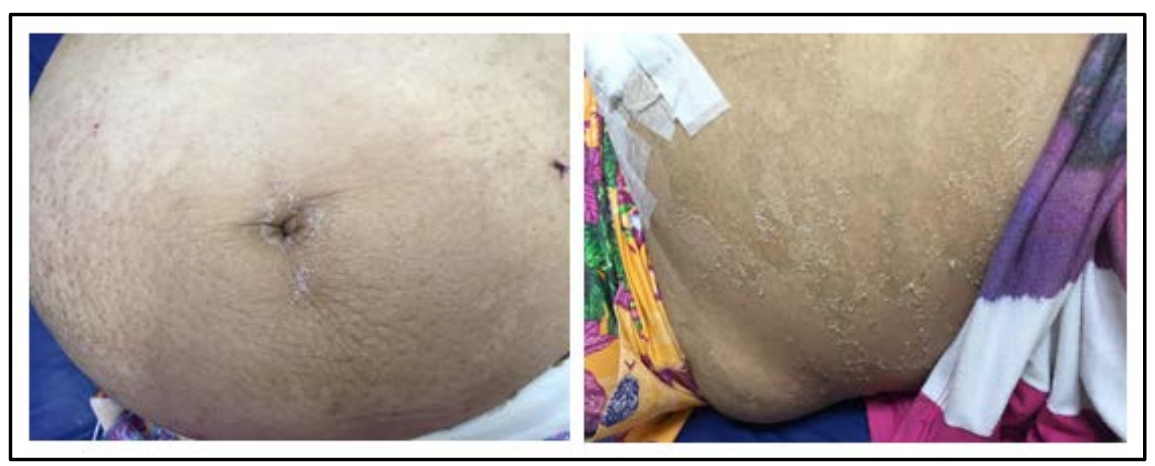

Figure 4. Resolution of the cutaneous reaction with presence of skin scaling on the 9th PO.

The anatomopathological examination of the skin biopsy showed alterations of discrete to moderate chronic perivascular dermatitis with presence of eosinophils, which are compatible with the diagnosis of AGEP (Figure 5).

\section{Discussion}

The diagnosis of dermatopathy by reaction to drugs is difficult, especially in patients who are receiving multiple drugs, and usually done by exclusion of other pathologies. In the presented case, the diagnostic hypothesis of AGEP was suspected by the persistence of febrile peaks and leukocytosis after the exclusion of multiple infectious foci (pulmonary, cutaneous and urinary), antibiotic therapy and surgical reintervention. Furthermore, the rapid development of new lesions after the administration of azithromycin, as well as its remission after discontinuation of this antibiotic, showed the relation of the pustular lesions with the use of azithromycin [7]-[12].

The etiology of AGEP is strongly associated with the use of antimicrobials, especially macrolides and beta-lactams, with a median of 24 hours from drug exposure to reaction onset, as observed in this case (1). Many other drugs may be related to the onset of lesions, such as quinolones, sulfonamides, terbinafine, antimalarials, calcium channel blockers and nonsteroidal anti-inflammatory 


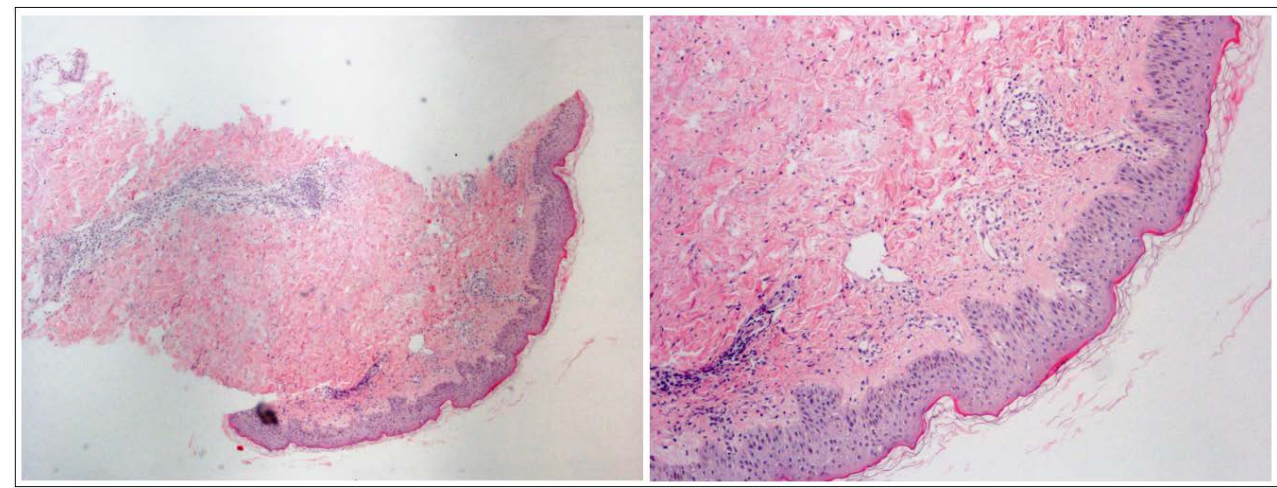

Figure 5. Anatomopathological examination of the skin biopsy.

drugs. In addition, there are other etiologies such as acute viral infections by Parvovirus B19, Cytomegalovirus, Coxsackievirus B4 and Mycoplasma pneumoniae [7]-[12].

As evidenced in this case report, the course of the disease is self-limiting, having simple and effective treatment, demonstrated by the significant improvement after the suspension of the causative drug. Symptomatic medications, such as antipyretics and corticosteroids, may be used according to the symptoms presented by the patients. In general, it has a good prognosis, except in elderly patients with persistent high fever or secondary infection of cutaneous lesions [11] [12].

As showed in another case report, a 71-year-old white woman presented with non-follicular pustules on oedematous erythema on the neck and in the antecubital folds. She had no fever or neutrophilia. Skin lesions began 1 day after she had finished a 3-day course of azithromycin. The patient was diagnosed with AGEP by skin biopsy and treated with symptomatic medications (topic clobetasol and loratadine once daily). As expected, the pustulosis cleared within 15 days from suspension of the azithromycin [13].

Pustular psoriasis is a diagnostic hypothesis to be considered in cases like this. Its pustules occur on top of an erythematous base, but it is slower in onset, and it uses to coalesce into large purulent collections. Generalized pustular psoriasis has been associated with pregnancy, drugs, and infection. There is often a personal or family history of psoriasis, which is not seen in AGEP, and it also differs in the histologic findings [1].

Pustulosis, which is identical to AGEP, can also be a first manifestation of a drug rash with eosinophilia and systemic symptoms (DRESS). Different from AGEP, the pustules usually appear after weeks of prior therapy and the disease manifests with Hepatitis or other internal involvement [6].

This case report reinforces the need to include AGEP as a differential diagnosis of dermatopathies observed during the use of medications, such as antibiotics. Despite its low incidence, the adverse reaction abruptly affects an extensive cutaneous area and leads to persistence of high fever, so it could be confused with a systemic infection and, consequently, treated in the wrong way. 


\section{Conclusion}

Acute Generalized Exanthematous Pustulosis is characterized by the acute and extensive onset of pustular lesions following the use of drugs, such as antibiotics, which usually has a good prognosis. However, the concomitance of fever and leukocytosis makes it necessary to exclude systemic infectious foci as well as other dermatological differential diagnoses. Therefore, clarification of the clinical picture is fundamental for the early diagnosis, since the suspension of the causative drug is necessary to resolve the condition.

\section{Conflicts of Interest}

The authors declare no conflicts of interest regarding the publication of this paper.

\section{References}

[1] Szatkowski, J. and Schwartz, R.A. (2015) Acute Generalized Exanthematous Pustulosis (AGEP): A Review and Update. Journal of the American Academy of Dermatology, 73, 843-848. https://doi.org/10.1016/j.jaad.2015.07.017

[2] Sidoroff, A. (2012) Acute Generalized Exanthematous Pustulosis. Chemical Immunology and Allergy, 97, 139-148. https://doi.org/10.1159/000335625

[3] Speeckaert, M.M., Speeckaert, R., Lambert, J. and Brochez, L. (2010) Acute Generalized Exanthematous Pustulosis: An Overview of the Clinical, Immunological and Diagnostic Concepts. European Journal of Dermatology, 20, 425-433.

[4] Sidoroff, A., Halevy, S., Bavinck, J.N.B., Vaillant, L. and Roujeau, J.-C. (2001) Acute Generalized Exanthematous Pustulosis (AGEP)-A Clinical Reaction Pattern. Journal of Cutaneous Pathology, 28, 113-119. https://doi.org/10.1034/j.1600-0560.2001.028003113.x

[5] Feldmeyer, L., et al. (2016) Acute Generalized Exanthematous Pustulosis: Pathogenesis, Genetic Background, Clinical Variants and Therapy. International Journal of Molecular Sciences, 17, 1214. https://doi.org/10.3390/ijms17081214

[6] Fernando, S.L. (2012) Acute Generalised Exanthematous Pustulosis. Australasian Journal of Dermatology, 53, 87-92. https://doi.org/10.1111/j.1440-0960.2011.00845.x

[7] Roujeau, J.-C., Bioulac-Sage, P., Bourseau, C., et al. (1991) Acute Generalized Exanthematous Pustulosis. Analysis of 63 Cases. Archives of Dermatology, 127, 1333-1338.

[8] Di Lernia, V. and Ricci, C. (2015) Fluconazole-Induced Acute Generalized Exanthematuous Pustulosis. Indian Journal of Dermatology, 60, 212.

[9] Criado, P.R., et al. (2004) Severe Cutaneous Adverse Drug Reactions: Relevant Aspects to Diagnosis and Treatment-Part II. Anais Brasileiros de Dermatologia, Rio de Janeiro, 79, 587-601.

[10] Sidoroff, A., Dunant, A., Viboud, C., et al. (2007) Risk Factors for Acute Generalized Exanthematous Pustulosis (AGEP) - Results of a Multinational Case-Control Study (EuroSCAR). British Journal of Dermatology, 157, 989-996.

[11] Razera, F., Olm, G.S. and Bonamigo, R.R. (2011) Dermatoses Neutrofílicas: Parte II. Anais Brasileiros de Dermatologia, Rio de Janeiro, 86, 195-211.

[12] Sousa, A.S., Lara, O.A.C.C., Papaiordanou, F., Marchioro, G.S.S. and Tebcherani, A. 
(2015) Acute Generalized Exanthematous Pustulosis x Von Zumbusch's Pustular Psoriasis: A Diagnostic Challenge in a Psoriatic Patient. Anais Brasileiros de Dermatologia, 90, 557-560.

[13] Campanon-Toro, M.V., et al. (2017) Acute Generalized Exanthematous Pustulosis (AGEP) Induced by Azithromycin. Contact Dermatitis, 76, 363-364. 